\title{
The $\beta$-tubulin monomer release factor (p14) has homology with a region of the DnaJ protein
}

\author{
Matxalen Llosa ${ }^{\mathrm{a}}$, Kerman Aloria ${ }^{\mathrm{a}}$, Rafael Campo ${ }^{\mathrm{a}}$, Rodolfo Padilla ${ }^{\mathrm{b}}$, Jesús Avila ${ }^{\mathrm{b}}$, \\ Luis Sánchez-Pulido ${ }^{c}$, Juan Carlos Zabala ${ }^{\mathrm{a}, *}$ \\ ${ }^{a}$ Departamento de Biología Molecular, Facultad de Medicina, Universidad de Cantabria, C. Herrera Oria s/n, 39011-Santander, Spain \\ ${ }^{\mathrm{b}}$ Centro de Biología Molecular, Facultad de Ciencias, Universidad Autónoma de Madrid (CSIC-UAM), Madrid, Spain \\ 'Protein Design Group, CNB-CSIC, Campus Universidad Autónoma de Madrid, Madrid 28049, Spain
}

Received 30 September 1996

\begin{abstract}
p14 is a molecular chaperone involved in $\beta$-tubulin folding which catalyzes the release of $\beta$-tubulin monomers from intermediate complexes. Here we demostrate that active p14 protein which we have purified from an overproducing Escherichia coli strain can also release $\beta$-tubulin monomers from tubulin dimers in the presence of an additional cofactor $(Z)$. Analysis of p14 secondary structure suggests that this protein may belong to a family of conserved proteins which share structural similarities with the J-domain of DnaJ. We have constructed deletions and site-directed mutations in the $p 14$ gene. A single D to $E$ mutation in the region shown in DnaJ to be an essential loop for its function affected the monomer-release activity of p14. These results support the hypothesis that this p14 loop interacts with $\beta$ tubulin in a similar fashion as Dna.J interacts with DnaK and suggest a possible role of p14 in the folding process.
\end{abstract}

Key words: Molecular chaperone; Protein folding; $\alpha$-Tubulin; $\beta$-Tubulin; J-domain; p14

\section{Introduction}

The mechanism by which proteins acquire their 3-dimensional conformation after ribosomal synthesis remains one of the greatest enigma of biology. Although all the information required for this process must be contained within the primary structure of any protein, there are many cases where certain proteins known as molecular chaperones are needed to assist the folding of others. There are several families of chaperones, two of which are exemplified by the heat shock proteins hsp70 and hsp60. The best studied examples are found in Escherichia coli: the GroEL/GroES chaperonine/cochaperonine, both forming toroidal multimers and the DnaK/ DnaJ chaperone/co-chaperone where DnaJ is a molecular chaperone itself $[1-4]$.

DnaJ is known to stimulate the ATPase activity of DnaK together with GrpE [5]. This stimulation correlates with an increased affinity of DnaK for certain substrates [6-8]. The $\mathrm{N}$-terminal 76 residues of DnaJ are responsible for physical interaction with DnaK and stimulation of its ATPase activity $[9,10]$. This segment which constitutes the most highly conserved region among the DnaJ family members has been termed the J-domain. Some DnaJ homologs only related by the presence of the J-domain are still capable of interacting with their cognate DnaK homolog. Also, all known single mutations affecting the DnaJ member/DnaK member interaction map in the $\mathbf{J}$-domain $[9,11,12]$. The tertiary structure of

*Corresponding author. Fax: (42) 201945.

E-mail: zabalajc(a)medi.unican.es this J-domain has been determined [13-16] although it is not yet available. This region contains a helix-turn-helix motif, the loop between the two helices containing the invariant residues HPD in all members of the family [17]. Mutations in any of these three residues abolish its activity [9-11]. Specifically, an $\mathrm{H}$ to $\mathrm{Q}$ DnaJ mutant shows a 5 -fold reduction in its DnaK binding ability [10].

The basic microtubule units are tubulin dimers which are formed from $\alpha$ - and $\beta$-tubulin monomers. Both $\alpha$ - and $\beta$ tubulin associate with multimolecular complexes before they can fold and incorporate into dimers [18]. Gao et al. [19] have described that two cellular cofactors are needed to obtain the functional tubulin dimers from $\mathrm{C}_{900}$ complexes. These have been named cofactors $\mathrm{A}$ and $\mathrm{B}$. Cofactor $\mathrm{B}$ has just been shown to be a mixture of three novel proteins, cofactors $\mathrm{C}$, $D$ and $E$ [20]. Cofactor A was purified to homogeneity from bovine testis [21]. Simultaneously, our group purified p14, a protein responsible for $\beta$-tubulin monomer release from $C_{300}$ complexes from pig testis [22]. The partial amino acid sequence obtained for p14 match only to that of cofactor A [22]. Thus, both proteins will be referred as pl4 hereafter.

pl4 has a monomer-release activity both from $\mathrm{C}_{900}$ [21] and from $\mathrm{C}_{300}$ [22]. Gao et al. (1994) cloned the mouse p14 cDNA and found no significant homologies to other known genes. These authors have suggested that pl4 is the CCT cochaperonine as it seems to increase the rate of ATP hydrolysis of the $\mathrm{C}_{900}$. However, it has not been possible to prove the direct interaction of p14 with CCT. In addition, Campo et al. (1994) have shown that pl4 can release monomers from the $\mathrm{C}_{300}$ complexes which apparently lack TCP-1 protein, and in the presence of ATPYS [23]. Thus, suggesting that this protein is a chaperone by itself. Recently, a yeast protein that shows $32 \%$ identity with mouse pl4 and binds to $\beta$-tubulin in vivo has been identified [24].

This study addresses the genetic and biochemical characterization of p14. We have purified the active protein from an overproducing $E$. coli strain and have analyzed the effect of several deletion and site-directed mutants on the monomerrelease activity of p14. We have found that p14 binds stably to $\beta$-tubulin monomers in vitro being this interaction probably mediated by a J-domain-like structure present in the p14 protein.

\section{Materials and methods}

\subsection{Plasmid constructions and protein purification}

Two synthetic oligonucleotides were used to replace the first 30 amino acids of p14. The rest of the p14 sequence was added by a PCR rcaction carried out using plasmid HHEA15G (Genexpress) and two other primers. Plasmid pMTX5 was generated and DNA se- 


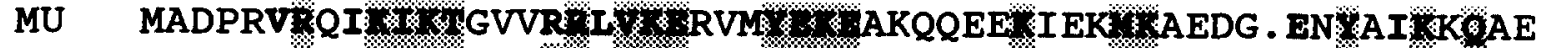

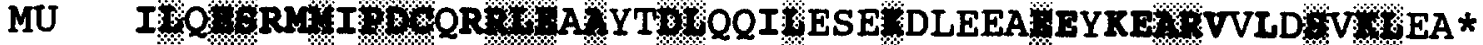

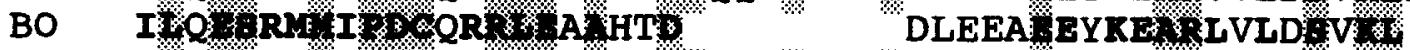

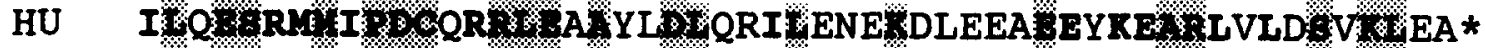

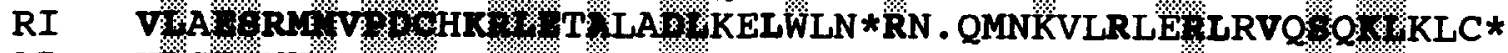 \\ AR VIG WWV

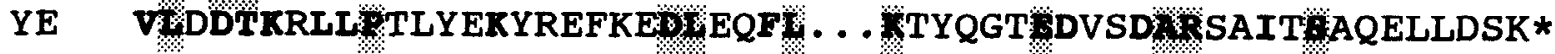

Fig. 1. The p14 protein family. Sequences were aligned using the Pileup facility of the GCG programme package (University of Wisconsin). These correspond to murine (MU) and bovine (BO) cofactor A [21], human (HU) p14 (EMBL accession numbers Z30197 and D20229), rice (RI) pl4 (accession number D15404), Arabidopsis (AR) pl4 (accession number T14083), and yeast (YE) Rbl2p [24]. Highly conserved or identical amino acids in all or all but one sequences are indicated with bold and shaded symbols, respectively. Dots denote gaps.

quenced. Stepwise deletions were carried out with exonuclease-III using the kit Erase-a-base (Promega). Plasmid pMTX5 was digested with $H$ indIII and incubated with exoIII at $30^{\circ} \mathrm{C}$. Aliquots were removed after $0.5,1,1.5$ and $2 \mathrm{~min}$ and were treated with S1 nuclease, the Klenow fragment of DNA polymerase I and DNA ligase. Plasmid pMTX5 was used as template to obtain p14 single mutants. Two PCR reactions were carried out with four different oligonucleotides. Mutations were determined by DNA sequencing. p14 wild-type and mutant proteins were purified essentially as previously described [22]. Samples were analyzed at each purification step by SDS-PAGE. Purified pl4 from pig testis [22] was used as a standard.

\subsection{Protein blotting}

Samples were loaded on either SDS-PAGE or native gels which were afterwards electrotransferred to nitrocellulose membranes (with $20 \%$ methanol in the transfer buffer for SDS-PAGE gels). Polyclonal anti-p 14 antibodies were raised by a 4 step injection of $200 \mu \mathrm{g} \mathrm{E}$. coli purified pl4 protein into rabbits. Injections were spaced 10 days. Mouse monoclonal anti- $\beta$ and anti- $\alpha$ tubulin antibodies were purchased from Amersham (N357 and N356, respectively). Bound primary antibodies were detected with horseradish peroxidase-conjugated sheep anti-mouse or donkey anti-rabbit secondary antibodies (Amersham NA931 and NA934, respectively), followed by ECL detection (Amersham).

\subsection{Monomer release assay from $C_{300}$ complexes}

Monomer release assays from $\mathrm{C}_{300}$ complexes were carried out esentially as previously described [22]. Reactions were electrophoresed in $7 \%$ non-denaturing polyacrylamide gels [18]. Monomer release assays from tubulin dimers were carried out as follows. Protein samples were incubated in $50 \mathrm{mM}$ MES, pH 6.7, containing $1 \mathrm{mM}$ GTP, $1 \mathrm{mM}$ $\mathrm{MgCl}_{2}$ for $90 \mathrm{~min}$ at $30^{\circ} \mathrm{C}$ in the presence or absence of $10 \mu \mathrm{g}$ FPLCpurified brain tubulin, $5 \mu \mathrm{g}$ purified p14 protein and partially purified cofactor Z. Reactions were loaded on non-denaturing $6 \%$ polyacrylamide gels. Resulting gels were either stained with Coomassie Brilliant Blue, or electrotransferred to nitrocellulose membranes for protein blotting.

\section{Results}

\subsection{The pl4 family of proteins}

The amino acid sequence of murine and bovine p14 [21] were used to search for homologous sequences in the Databases using the Tfasta facility of the GCG package of programmes (University of Wisconsin [25]). We found two human partial cDNAs (both lacking the $5^{\prime}$ end), a partial cDNA from Arabidopsis thaliana ( $3^{\prime}$ end missing), and a complete
cDNA from rice that coded for peptides homologous to the known p14 sequences (Fig. 1). The human p14 segment showed greater than $92 \%$ identity with murine and bovine pl4; the pl4 from rice shared almost $82 \%$ identity with the p14 segment from Arabidopsis; and, finally, p14s from animals were about $35 \%$ identical and $60 \%$ homolog to those described for plants. These homologies strongly suggested a close relationship among this group of proteins that probably reflected a common physiological role in all superior plants and animals. In addition, p14 proteins showed about 30\% identity with yeast Rbl2p protein [24].

\subsection{Overexpression and purification of p14 protein}

A partial human cDNA named HHEA1SG (EMBL accession \#Z30197) from an atrium Genexpress cDNA library was obtained and sequenced. The resulting sequence is shown in Fig. 1. This clone was missing the 5 ' sequence which presumably coded for the first 30 amino acids of human p14, according to the sequence alignment shown in Fig. 1. This gene was

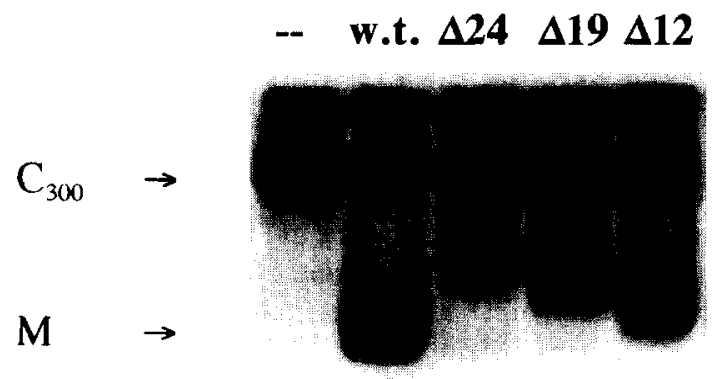

Fig. 2. Monomer-release activity of pl4 deletion derivatives. Fluorography of a non-denaturing $7 \%$ polyacrylamide gel showing the release of labelled tubulin from $\mathrm{C}_{300}$ intermediate complexes, in the presence of different p14 deletion derivatives. Reactions contained $10 \mu \mathrm{l}$ of supernatants from IPTG-induced BL21:DE3 containing plasmids (from left to right) pET-3a, pMTX5 (p14), pMTX6

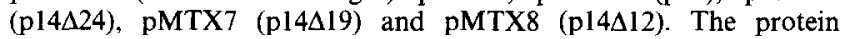
overproduced is indicated on the top of each lane. The position of the $C_{300}$ complexes and the tubulin monomer species (M) as previously described (see text) are indicated by the arrows drawn on the left. 
C. B.

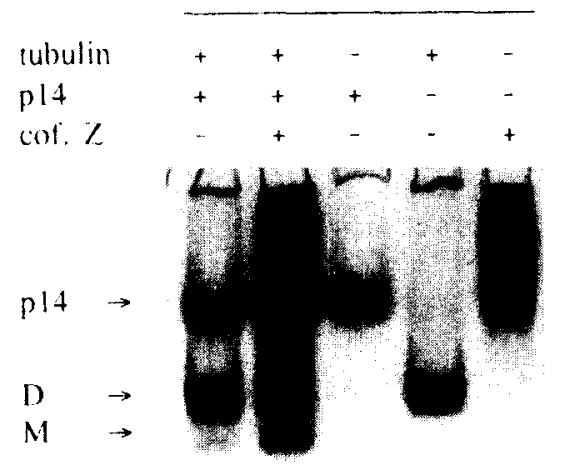

anti-p14

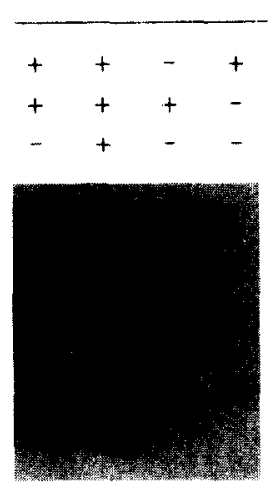

anti-ß

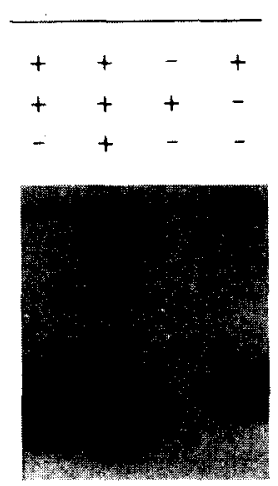

anti- $\alpha$

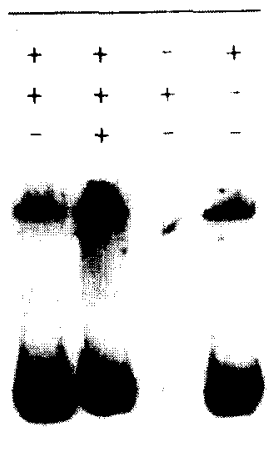

Fig. 3. $\beta$-Tubulin monomer release depends upon p14 binding. Monomer-release reactions from tubulin dimers were loaded onto non-denaturing $6 \%$ polyacrylamide gels. (C.B.), Coomassie Brilliant Blue stained gel similar to that immunostained in the next three panels (anti-p14, anti$\beta$ and anti- $\alpha$ panels). Next three panels: Western blot analysis of the same reactions immunoprobed sequentially with the antibodies indicated above each gel revealing the position of the p14 dimer (p14), the tubulin heterodimer (D) and the p14/ $\beta$-tubulin monomer complex (M), all indicated by arrows on the far left of the figure.

reconstructed by adding the missing sequence with synthetic oligonucleotides coding for the first 30 amino acids of murine p14. To purify p14 from the $E$. coli overproducing strain we adapted the protocol of Campo et al. (1994). Overproduced protein which was mainly accumulated in inclusion bodies in $E$. coli was solubilized with $8 \mathrm{M}$ urea. The obtained mixture was sequentially chromatographed through a DEAE-cellulose column a HiTrap Blue column and finally a Superdex $75 \mathrm{HR}$ gel filtration column. The resulting protein was electrophoretically pure and retained its monomer-release activity in all steps.

Pure p14 protein was used to produce antibodies as described, which were found highly specific. p14 was found to be expressed in all examined tissues although at significantly higher amounts in testis. These results agreed with those obtained for p14 mRNA [21]. Immunofluorescence localization of pl4 performed with the same antibodies demostrated the protein distributed throughout the cell cytoplasm though not colocalized with microtubules (data not shown).

\subsection{Stepwise deletions in the p14 gene}

In a first attempt to determine the p14 functional motifs we carried out exolII-deletions of the p14 gene from the $3^{\prime}$ end. Three plasmids were selected (pMTX6, pMTX7 and pMTX8) that overproduced in BL21:DE3 visible proteins of smaller sizes than wild-type p14. These mutant proteins were named p14 $\Delta 24$, p14 19 and pl4 12 , respectively, referring to the number of carboxy-tcrminal p14 rcsidues deleted in each casc.

We assayed the monomer-release activity of the lysates from BL21:DE3 cells harbouring each of these plasmids (Fig. 2). All of them showed a reduced activity when compared to BL21:DE3(pMTX5); although it has to be noted that wild-type pl4 is overproduced in higher amounts (data not shown). Released monomers run in a different position in the native gels being retarded when compared to the p14-released monomers. This was more obvious in the case of supernatants containing p14 $\Delta 19$ and p14 $\Delta 24$. These p14 derivatives had a net charge of +4 and +6 , respectively, when compared to the wild-type p14, while p14 412 had the same net charge as $\mathrm{p} 14$, although a different size. These results suggested that the released species correspond to $\mathrm{p} 14 / \beta$-tubulin monomer complexes.

\section{4. $p 14$ releases $\beta$-tubulin monomers from tubulin dimers}

Since p14 has $\beta$-tubulin monomer binding ability in vivo (as shown for its homolog Rbl2p) we investigated whether p14 could also release monomers from tubulin dimers in vitro. We have found that p14 can also release $\beta$-tubulin monomers from purified tubulin heterodimers in the presence of another cofactor which we have called cofactor $Z$. When both p14 and cofactor $\mathrm{Z}$ are incubated with purified tubulin, released monomers are clearly visible in native gels (Fig. 3, panel C.B.) running at the same position as the labelled monomers released from the $C_{300}$ assay in the native gels (not shown). Cofactor $\mathrm{Z}$ has already been partially purified and its characterization will be described elsewhere.

Aliquots of the same reactions shown in panel C.B. were loaded in another gel, run and electrotransferred to a nitrocellulose membrane and sequentially blotted with various antibodies (Fig. 3). The released species corresponded to $\beta$ tubulin monomers bound to p14 while $\alpha$-tubulin was found present only in the dimers. The amount of $\alpha$-tubulin was higher when no $\beta$-monomeric forms were released.

The size of the p14/ $\beta$-tubulin complex was determined by gel filtration analysis obtaining a value of $60 \mathrm{kDa}$. In order to characterize the content of the p14 $\beta /$ tubulin band in native gels, this band was excised and analyzed in a SDS-polyacrylamide gel. This analysis indicated stoichiometric amounts of $\beta$-tubulin and p14 polypeptides (not shown) thus, suggesting that single $\beta$-tubulin monomers were bound to single p14 molecules.

\subsection{Structural similarity to the J-domain}

Detailed p14 amino acid sequence and secondary structure analyses suggested a similarity to the J-domain of the DnaJ protein showing a region with a putative helix-turn-helix structure (Fig. 4). The network of apolar residues in the internal face of the $\alpha$-helices was remarkably similar to some known $\alpha-\alpha$ interfaces. Particularly, two sequence patterns $(\mathrm{I} / \mathrm{L} \mathrm{R} / \mathrm{K} / \mathrm{L} \mathrm{K} \times \mathrm{x} \times \mathrm{x} / \mathrm{A} / \mathrm{V}$ and $\mathrm{L} \times \mathrm{A} / \mathrm{S} / \mathrm{F} \mathrm{Y} / \mathrm{H} / \mathrm{L} / \mathrm{K} \times \mathrm{x}$ L) were also found as part of the contact between $\alpha$-helices in DnaJ domains [13-16] and G-CSF proteins (cytokine granulocyte colony stimulating factor [26]). In both cases, despite their different topologies, the pattern of interactions between the two regions was highly conserved with some very well 

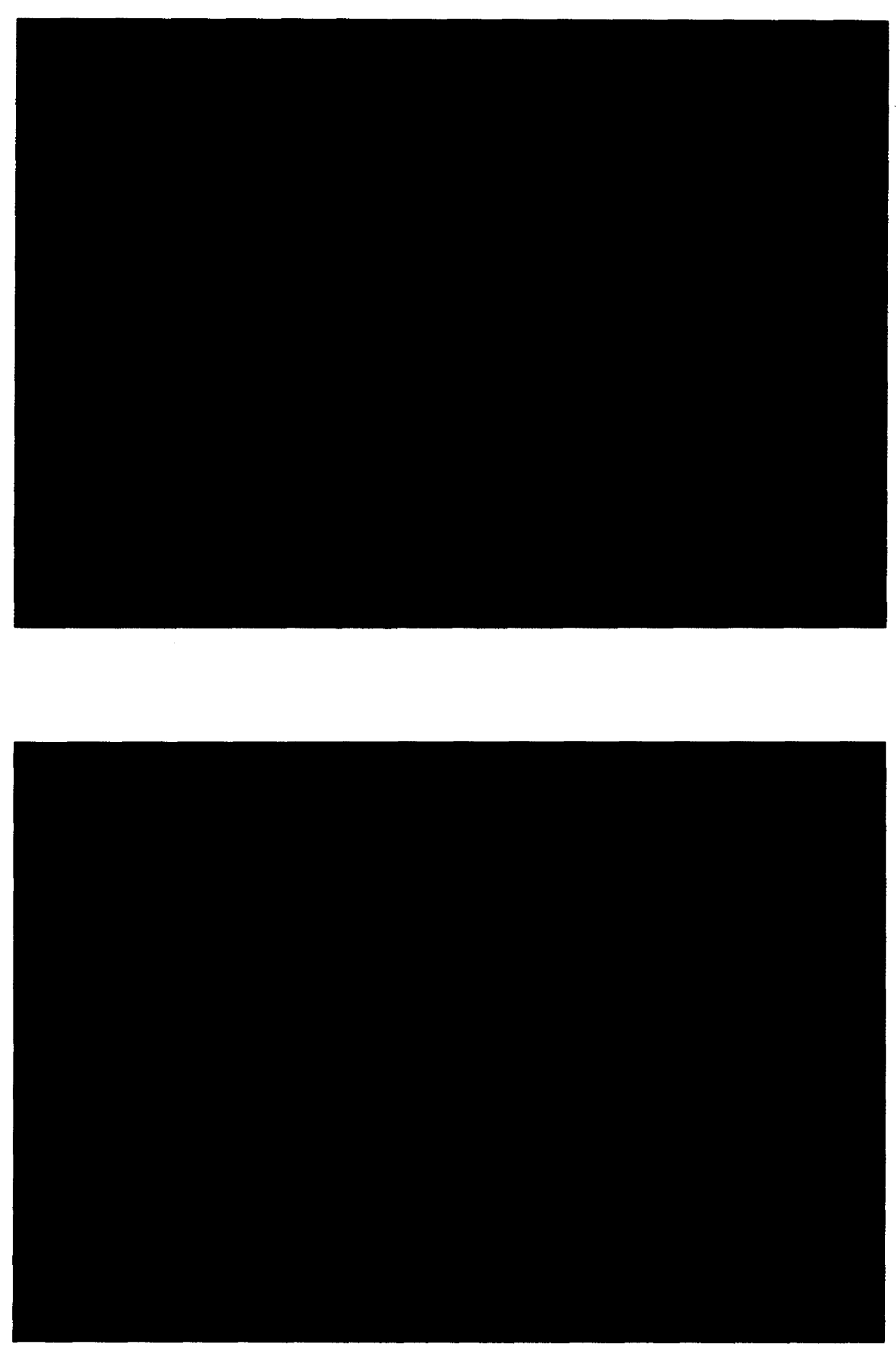

preserved key interactions (see Fig. 4). It is quite possible that p14 resembles these structures with two $\alpha$-helices interacting in anti-parallel fashion and contacting strongly at the level of the two sequence patterns. The presence of the conserved PD sequence found in Dna.J responsible for the formation of the loop between the two $\alpha$-helices and the computer experiment of replacing the residues of p14 in the structure of the G-CSF showing a good degree of adaptation supported the proposed 
Fig. 4. Proposed structure of p14. $\alpha-\alpha$ interface similarity with the J-domain of DnaJ. Stereo view of the Csf3 fragment structure (PDB entry: $1 \mathrm{bgc}$ ). Contacting residues are shown with thick sticks (upper figure). Some selected DnaJ sequences were aligned to the known p14 sequences in the helix-turn-helix region. In the bottom figure some selected sequences of the G-CSF family were also indicated. The position of the $\alpha$-helices in the structure of DnaJ are shown on the top figure. These correspond with the predicted secondary structure of p14 and the structure of Csf3 [26,36]. The most important contacts between the $\alpha$-helices that are present in the structure of DnaJ [13-16] and Csf3 [26] are shown in the lowest figure as lines connecting the corresponding residues. The DnaJ and Csf3 sequences are labelled with their database codes [37]. p14 sequences coincide with those in Fig. 1. $\leftarrow$

structural p14 prediction. The quality of the resulting structures was assessed by three different standard structure checking methods after the modelling. One of the methods was based on normality of molecular contacts, and the other two in deviation from normal exposed hydrophobic surfaces [27-29]. In all three cases the obtained values were in the range of normal globular proteins being very similar to the values of the native proteins.

There are two experimental data which additionally support the above hypothesis. First, when p14 from pig testis was subjected to proteolysis with trypsin [22], the peptide MMIPD (including the conserved PD residues) was obtained, as expected for an exposed loop structure. And second, we have determined the circular dichroism spectrum of p14 and have found similarities to that corresponding to the J domain [14]. Both spectra show large negative peaks of ellipticity at 208 and $222 \mathrm{~nm}$ and a large positive peak at $195 \mathrm{~nm}$ indicating a relative large content of $\alpha$-helix.

\subsection{Site-directed mutants of p14}

Site-directed p14 gene mutants were designed in order to test further the proposed structural model. These were the Asp-66 (conserved both among the pl4s and the DnaJs) to Glu; and the Cys-67 (common to all pl4s except for Rbl2p) to Ser.

Limiting amounts of wild-type and mutant proteins (p14D66E and p14C67S) were tested for their monomer-release activity from labelled $\mathrm{C}_{300}$ complexes (Fig. 5) where the half maximum ligand binding $\left(\mathrm{L}_{1 / 2}\right)$ concentration was calculated. This factor was expected to change in those cases where the mutated region would be implicated in the interaction with $\beta$-tubulin. We found that the $\mathrm{C} 67 \mathrm{~S}$ mutation induced an increased monomer-release activity while the D66E mutation impaired this activity (Fig. 5), thus suggesting that this p14 protein region was involved in tubulin binding.

\section{Discussion}

p14 protein was originally purified from pig testis as a tubulin monomer-release factor from $\mathrm{C}_{300}$, the intermediates tubulin folding complexes. Its physiological role became definitely associated to tubulin when the p14 homolog Rbl2p from yeast was found to bind to $\beta$-tubulin in vivo [24]. Now we have found that there is a family of homologous proteins which includes the previously described cofactor A from mouse and calf, $\mathrm{Rbl} 2 \mathrm{p}$ from yeast, as well as other previously unidentified proteins belonging to man and plants (Fig. 1). It seems likely that in view of the high level of homology between all these proteins they may play the same physiological role.

In order to address a detailed genetic and biochemical analysis of p14 protein, we have constructed a chimaeric murine/ human 14 gene, and purified the overproduced protein from
E. coli. The obtained protein did not show any functional difference in vitro compared to that purified from pig testis. This purification protocol included a strong treatment with urea after which native pl4 was generated by in vitro folding techniques.

The C-terminal end of p14 is not apparently essential for its function since the deletion derivatives $\Delta 12, \Delta 19$ and $\Delta 24$ retained the monomer release activity. However, these reactions were less efficient than those using wild-type p14, suggesting a specific role of the carboxy-terminal domain in monomer release. Apparently, the removal of the C-terminal 12 residues affects p14 activity as much as removal of the C-terminal 24 residues. The most interesting result from the deletion derivatives of p14 was the altered mobility of the molecular species released from the $\mathrm{C}_{300}$ complexes (Fig. 2). This fact suggested that this species were not merely a $\beta$-tubulin monomer but most probably a p14/ $\beta$-tubulin complex, since the level of retardation in the gel corresponded with the increasing isoelectric point of the p14 C-terminal deletion derivatives.

We have found that $\mathrm{p} 14$ releases $\beta$-tubulin monomers also from purified tubulin dimers (Fig. 3) requiring an additional cellular cofactor(s) named cofactor $Z$ that is only partially purified yet. We have showed by immunoblots the presence

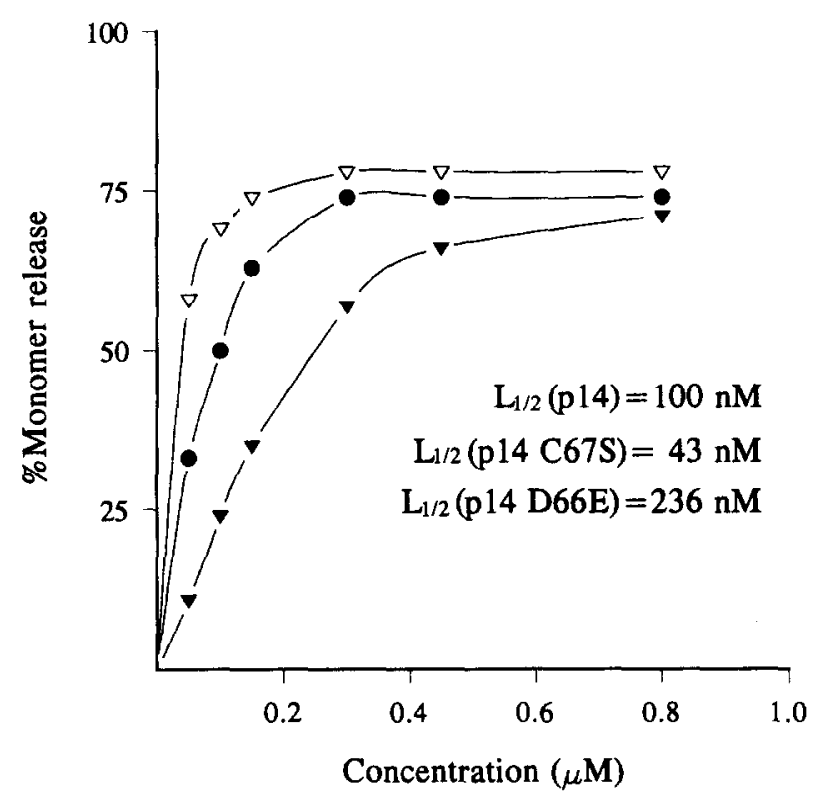

Fig. 5. Monomer-release activity of pl4 site-directed mutants. Monomer-release assays from labelled $\mathrm{C}_{300}$ complexes were performed under limiting protein amounts and electrophoresed under non-denaturing conditions. Bands were quantified using a BioRad GS-363 Molecular Imager System after fluorography of the gel. This figure shows the average of the percentage of label in the monomeric form released. $\bullet$, Wild-type p14; $\nabla, \mathrm{p} 14 \mathrm{C} 67 \mathrm{~S} ; \nabla, \mathrm{p} 14$ D66E. Half-maximum binding constant $\left(L_{1 / 2}\right)$ is defined as the amount of protein required to release $50 \%$ of the labelled $\beta$-tubulin from the complexes. 
of both p14 and $\beta$-tubulin in the species released from tubulin dimers (Fig. 3). These results support in vitro the in vivo observation that the yeast homolog Rbl2p binds to $\beta$-tubulin [24]. Our results indicate that a single p14 molecule binds to a single $\beta$-tubulin molecule, thus agreeing with other authors hypothesis suggesting that $\mathrm{Rb} 2 \mathrm{p}$ compensates for the defects associated with either too much $\beta$-tubulin or too little $\alpha$-tubulin. In addition to the active role of p14 in the tubulin folding pathway this protein could work as a 'sequestering' factor of $\beta$-tubulin monomers. Thus, maintaining the equilibrium between $\alpha$ - and $\beta$-tubulin and preventing a lethal excess of $\beta$-tubulin in the cell [30-32].

A computer-assisted structural analysis of pl4 and database comparisons have revealed that this protein family is structurally related to the $\mathrm{J}$-domain present in the DnaJ protein family. The pl4s show a putative helix-turn-helix region similar to that of the DnaJs or the G-CSFs (Fig. 4). To reinforce the significance of the similarity based on the sequence similarity we have computer-replaced the sequence of $\mathrm{Csf} 3$ in this region for the corresponding consensus sequences of DnaJ or p14, obtaining values that were very similar to those for native proteins. The fact that both $\mathrm{p} 14$ and the J-domain show similar circular dichroism spectra supports additionally the above hypothesis.

This hypothesis led us to construct p14 mutants in the proposed loop rcgion and to assay these for their monomerrelease activity from labelled $\mathrm{C}_{300}$ complexes. Obtained results showed that although neither Asp-66 nor Cys-67 were essential for p14 function both residues were involved in $\beta$-tubulin interaction, since highly conservative mutations in both of them alter the $\mathrm{L}_{1 / 2}$ value (Fig. 5). These results confirm that the p14 similarity to DnaJ is not only structural as it is with G-CSF. Interestingly, the monomer-release activity is increased for the C67S mutant and was reduced for the D66E mutant which could indicate that the presence of $\beta$-turn formers (i.e., Ser or Asp; [33]) may increase p14 activity. On the other hand, it has to be noted that Cys-67 has no obvious counterpart in the DnaJ sequence while Asp-66 is highly conserved among both p14- and DnaJ-protein families except for yeast Rbl2p (Fig. 4). Furthermore, mutations in the homologous Asp residue of the DnaJ-homolog Sec63 result in an inactive protein [11]. Asp-66 could be directly involved in $\beta$ tubulin interaction as the corresponding region in DnaJ is assumed to be involved in DnaK interaction.

DnaJ and more precisely its $\mathrm{J}$-domain region stimulates the rate of hydrolysis of the DnaK-bound ATP $[5,9,10]$. We have previously shown that GTP hydrolysis is required to get tubulin monomers incorporated into dimers [23]. Thus, it is plausible to suggest that p14 would stimulate the GTPase activity of $\beta$-tubulin during the folding process concluding with the formation of the tubulin dimer.

During the preparation of this manuscript Melki et al. [34] published the functional and structural characterization of cofactor A. They have shown that cofactor A is a monomeric protein which gives a CD spectrum identical to that of $\mathrm{p} 14$. Also, they have found that cofactor A participates in the tubulin folding process by interacting with a folding intermediate of $\beta$-tubulin that is released from CCT complexes confirming our previous results $[22,23,35]$.

We would finally like to note that p14 is the only known protein apart from $\alpha$-tubulin that binds stably to $\beta$-tubulin. We hope that the isolation and characterization of $\mathrm{p} 14 / \beta$-tu- bulin complexes and cofactor $\mathrm{Z}$ will help to the purification of large amounts of $\beta$-tubulin monomers in the nearest future, thus contributing to the so far hampered structural studies of this fundamental protein.

Acknowledgements: We are very grateful to Dr. B. Obermaier-Skrobranek from Genexpress for having kindly providing us with the HHEA15G plasmid; to L. Serrano (EMBL) for determining the circular dichroism spectrum of pl4; to A. Valencia (Protein Design Group) for assessment on secondary structure analysis; and to M.L. Fanarraga, F. de la Cruz and J.M. García-Lobo for helpful and stimulating discussions. K.A. is a recipient of a predoctoral fellowship from the Eusko Jaurlaritza. This work was supported by a grant (PB94-1064) from the DGICYT to J.C.Z.

\section{References}

[1] Craig, E.A., Weissman, J.S. and Horwich, A.L. (1994) Cell 78, 365-372.

[2] Ellis, R.J. and van der Vies, S.M. (1991) Annu. Rev. Biochem. $60,321-347$.

[3] Ilendrick, J.P. and Hartl, F.U. (1993) Annu. Rev. Biochem. 62, 349-384.

[4] Morimoto, R.I., Tissieres, A. and Georgopoulos C. (1994) in: The Biology of Heat Shock Proteins and Molecular Chaperones (Morimoto, R.I., Tissieres, A. and Georgopoulos, C., eds.), pp. 1-30, Cold Spring Harbor Laboratory Press, Cold Spring Harbor, NY.

[5] Liberek, K., Marszalek, J., Ang, D., Georgopoulos, C. and Zylicz, M. (1991). Proc. Natl. Acad. Sci. USA 88, 2874-2878.

[6] Liberek, K. and Georgopoulos, C. (1993) Proc. Natl. Acad. Sci. USA 90, 11019-11023.

[7] Wickner, S., Hoskins, J. and McKenney, K. (1991) Nature 350, $165-167$.

[8] Schröder, H., Langer, T., Hartl, F.-U. and Bukau, B. (1993) EMBO J. 12, 4137-4144.

[9] Wall, D., Zylicz, M. and Georgopoulos, C. (1994) J. Biol. Chem. $269,5446-5451$.

[10] Wall, D., Zylicz, M. and Georgopoulos, C. (1995) J. Biol. Chem. 270, 2139-2144.

[11] Feldheim, D., Rothblatt, J. and Schekman, R. (1992) Mol. Cell. Biol. 12, 3288-3296.

[12] Nelson, M.K., Kurihara, T. and Silver, P.A. (1993) Genetics 134, $159-173$.

[13] Szyperski, T., Pellecchia, M., Wall, D., Georgopoulos, C. and Wüthrich, K. (1994) Proc. Natl. Acad. Sci. ISA 91, 1134311347.

[14] Hill, R.B., Flanagan, J.M. and Prestegard, J.H. (1995) Biochemistry 34, 5587-5596.

[15] Qian, Y.Q., Patel, D., Hartl, F.-U. and McColl, D.J. (1996) J. Mol. Biol. 260, 224-235.

[16] Pellecchia, M., Szyperski, T., Wall, D., Georgopoulos, C. and Wüthrich, K. (1996) J. Mol. Biol. 260, 236-250.

[17] Kelley, W.L. and Landry, S.J. (1994) Trends Biochem. Sci. 19, 277-278.

[18] Zabala, J.C. and Cowan, N.J. (1992) Cell Motil. Cytoskel. 23, 222-230.

[19] Gao, Y., Vainberg, I.E., Chow, R.L. and Cowan, N.J. (1993) Mol. Cell. Biol. 13, 2478-2485.

[20] Tian, G., Huang, Y., Rommelaere, H., Vandekerckhove, J., Ampe, C. and Cowan, N.J. (1996) Cell 86, 287-296.

[21] Gao, Y., Melki, R., Walden, P.D., Lewis, S.A., Ampe, C., Rommelaere, H., Vandekerckhove, J. and Cowan, N.J. (1994) J. Cell Biol. 125, 989-996.

[22] Campo, R., Fontalba, A., Sánchez, L.M. and Zabala, J.C. (1994) FEBS Lett. 353, 162-166.

[23] Fontalba, A., Paciucci, R., Avila, J. and Zabala, J.C. (1993) J. Cell Sci. 106, 627-632.

[24] Archer, J.E., Vega, L.R. and Solomon, F. (1995) Cell 82, 425434.

[25] Devereux, J., Haeberli, P. and Smithies, O. (1984) Nucl. Acids Res. 12, 387-395.

[26] Lovejoy, B., Cascio, D. and Eisenberg, D. (1993) J. Mol. Biol. $243,640-653$. 
[27] Holm, L. and Sander, C. (1992) J. Mol. Biol. 225, 93-105.

[28] Vriend, G. (1990) J. Mol. Graph. 8, 52-56.

[29] Vriend, G. and Sander, C. (1993) J. Appl. Cryst. 26, 47-60.

[30] Burke, D., Gasdaska, P. and Hartwell, L. (1989) Mol. Cell. Biol. 9, 1049-1059.

[31] Katz, W., Weinstein, B. and Solomon, F. (1990) Mol. Cell. Biol. $10,2730-2736$.

[32] Weinstein, B. and Solomon, F. (1990) Mol. Cell. Biol. 10, 52955304.
[33] Chou, P.Y., and Fasman, U.D. (1974) Biochemistry 13, 222-245.

[34] Melki, R., Rommelaere, H., Leguy, R., Vandekerckhove, J. and Ampe, C. (1996) Biochemistry 35, 10422-10435.

[35] Zabala, J.C., Fontalba, A. and Avila, J. (1996) J. Cell Sci. 109, $1471-1478$.

[36] Rost, B. and Sander, C. (1993) J. Mol. Biol. 232, 584-599.

[37] Bairoch, A. and Boeckmann, B. (1993) Nucl. Acids Res. 21, 3093-3096. 